\title{
Altered Mitochondrial Function and Cystic Fibrosis
}

\section{Awasthi $\mathrm{A}^{1 *}$, Prasad $\mathrm{B}^{2}$ and Kumar $\mathrm{J}^{3 *}$}

${ }^{1}$ Department of Biology, School of Engineering, Presidency University Bangalore, 560089, Karnataka, India

${ }^{2}$ Department of Botany/Biotechnology, Patna University, Patna, 800005, Bihar, India

${ }^{3}$ DBT-PU-IPLS Programme, Department of Botany/Biotechnology, Patna University, Patna, P.O. Box: 800005, Bihar, India

\begin{abstract}
Cystic fibrosis (CF) is an autosomal recessive disorder caused by mutation in cystic fibrosis transmembrane conductance regulator gene (CFTR). CFTR is a membrane glycoprotein which functions as cyclic adenosine monophosphate (CAMP) activated anion channel. Mutation in CFTR effect's its synthesis, processing, regulation and function resulting in CF. Mutated CFTR results in dysregulation of fluid and electrolyte transport across epithelial cell membranes. Mutated CFTR has also been implicated to alter mitochondrial structure and function. All the probable mitochondrial functions like components of electron transport chain, calcium buffering, reactive oxygen species generation, levels of mitochondrial glutathione have been implicated to be effected in CF. CFTR has also been reported to regulate functioning of two mitochondrial genes CISD1 and MT-ND4. All the reports illustrating the role of $\mathrm{CF}$ in altering mitochondrial function are scrutinized and presented in this review.
\end{abstract}

Keywords: Cystic fibrosis; Mitochondria; CFTR; MT-ND4; CISD1; Calcium; Glutathione

Abbreviations: CF: Cystic Fibrosis; CFTR: Cystic Fibrosis Transmembrane Conductance Regulator; ABC: ATP-Binding Cassette; MSD: Membrane Spanning Domains; NBD: Nuclear Binding Domain; GSH: Glutathione Stimulating Hormone; GR: Glutathione Reductase; ROS: Reactive Oxygen Species

\section{Introduction}

Cystic fibrosis (CF) is an autosomal recessive multisystem disorder $[1,2]$. It is caused by mutation in cystic fibrosis transmembrane conductance regulator gene (CFTR) [1-3]. CFTR is a membrane protein which acts a channel for transport of chloride ions [3]. Transport of chloride ions regulate water and salt content of the epithelial surface resulting in thin mucus formation, predominantly in respiratory, digestive and reproductive tissues [1-5]. Disruption of ion transport due to mutated CFTR gene results in production of thick viscous mucous and increased salt concentration in the sweat (Figure 1) [1-5]. It results in blockage of the airways, ducts, glands resulting in complications like pulmonary failure, gastrointestinal disorders as well as infertility [1-6].

CFTR is a $170 \mathrm{kDA}$ membrane glycoprotein belonging to ATPbinding cassette $(\mathrm{ABC})$ transporter gene super family [7]. CFTR protein has two membrane spanning domains (MSD), made up of six helices each which are connected to the nuclear binding domain (NBD 1 and

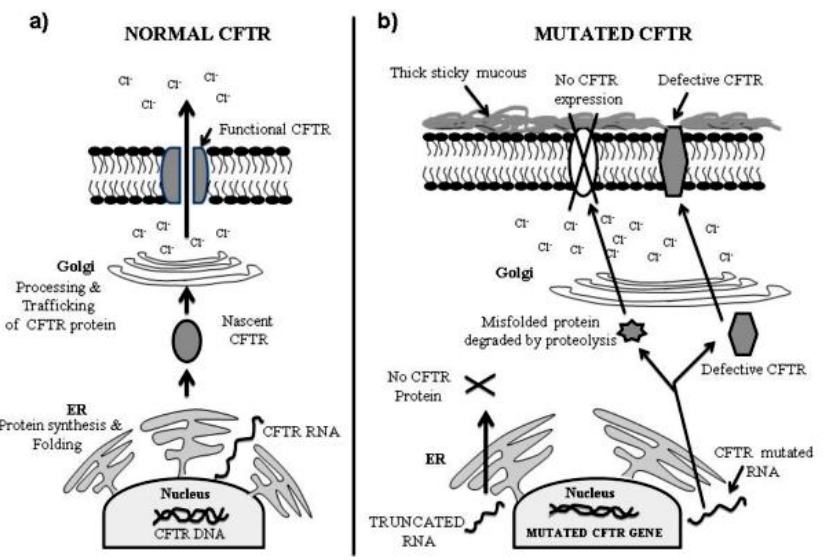

Figure 1: Role of CFTR mutation in cystic fibrosis. a) Normal CFTR channel expression facilitates chloride $\left(\mathrm{Cl}^{-}\right)$movement across the plasma membrane resulting in thin watery mucus. b) Mutated CFTR disrupts $\mathrm{Cl}^{-}$ion movement resulting in thick viscous mucous.

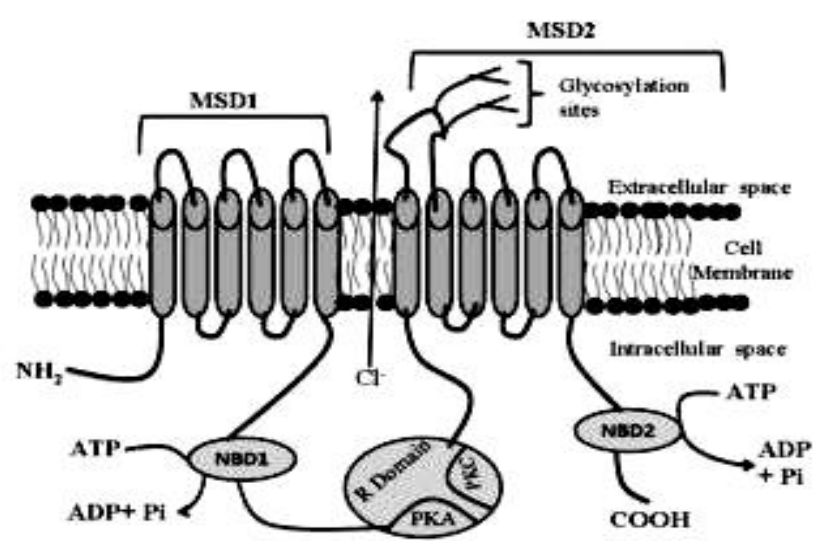

Note: MSD: Membrane Spanning Domain; NBD: Nuclear Binding Domain; R Domain: Regulatory Domain; PKA: Protein Kinase A; PKC: Protein Kinase C.

Figure 2: Structure of CFTR protein.

2). NBD's are involved in ATP binding and hydrolysis [7]. In between NBD 1 and MSD2 is present regulatory (R) domain. The R domain contain sites for cyclic adenosine monophosphate (cAMP) dependent phosphorylation substrates for protein kinase A and C. ATP binding to $\mathrm{NBD}$ and phosphorylation of $\mathrm{R}$ domain regulates ion channel activity of CFTR (Figure 2) [7]. Around 1997 CFTR gene mutations are listed in the cystic fibrosis mutation database (http://www.genet.sickkids. on.ca). Deletion of phenylalanine at position 508 (F508del-CFTR), is the most predominant mutation reported to be associated with $\mathrm{CF}$ and it is present in NBD 1 [8].

Mutated CFTR causes defective intracellular transport resulting

*Corresponding authors: Dr. Anjali Awasthi, Department of Biology, School of Engineering Presidency University Bangalore, 560089, Karnataka, India, Tel: 01596245073; E-mail: anjaliaw@gmail.com

Dr. Jitendra Kumar, DBT-PU-IPLS Programme, Department of Botany/ Biotechnology, Patna University, Patna, 800005, Bihar, India, Tel: 01596245073; E-mail: jitendra343@gmail.com

Received May 15, 2015; Accepted August 05, 2013; Published August 08, 2015

Citation: Awasthi A, Prasad B, Kumar J (2015) Altered Mitochondrial Function and Cystic Fibrosis. Genetics S7: 001. doi:10.4172/2161-1041.S7-001

Copyright: $\odot 2015$ Awasthi A, et al. This is an open-access article distributed under the terms of the Creative Commons Attribution License, which permits unrestricted use, distribution, and reproduction in any medium, provided the original author and source are credited. 
in CF etiology. Role of defective CFTR in altering mitochondrial functioning has been reported. All the available literature illustrating alteration of mitochondrial functioning in cystic fibrosis has been reviewed and accounted in the underlying sections.

\section{Mitochondrial function and cystic fibrosis: Evidence in 1970's and 1980's}

The role of altered mitochondrial function in CF was hypothesised as early in 1979 by Burton L. Shapiro et al. [9-13]. Using cultured fibroblast cells from the CF patients and control they have reported that $\mathrm{CF}$ cells showed more oxygen consumption compared to the control, moreover inhibition of the mitochondrial electron transport system using rotenone resulted in distinctive rates of inhibition of oxygen consumption in control and CF cells [10]. Nicotinamide adenine dinucleotide (NADH) dehydrogenase, which is the enzyme of complex I of the mitochondrial electron transport system also showed differences in enzyme kinetics with decreased $\mathrm{Km}$ and increased $\mathrm{pH}$ optima in CF cells [9-14]. Similarly, altered kinetics for cytochrome-c oxidase resulting in increased $\mathrm{Km}$ at temperature $>25^{\circ} \mathrm{C}$ have been reported in CF fibroblast [15].

CF cells were also reported to show increase in the intracellular calcium concentration compared to control [16-18], moreover mitochondria showed enhanced calcium uptake [18]. They have also reported an increase in relative electron transport activity of mitochondrial complex in case of $\mathrm{NADH}$-oxidase, $\mathrm{NADH}$-cytochrome $c$ reductase, and succinate-cytochrome $\mathrm{c}$ reductase in $\mathrm{CF}$ cells compared to control [18]. Altered levels of glutathione (GSH) and activity of glutathione reductase (GR) has been reported in CF compared to control $[19,20]$. Altered levels of GSH are implicated in the conditions of oxidative stress, moreover, mitochondria are one of the prime sites for oxidative stress and GSH plays a critical role in maintaining redox homeostasis. All these lines of evidences reported in late 1970's and 1980's supported the role of mitochondrial dysfunction in CF.

\section{Mitochondrial dysfunction and cystic fibrosis: Post 1990's}

Advent of cloning technique facilitated cloning of CF gene in late 1980 's and revealed it as a chloride channel $[3,4]$. Further research facilitated that CFTR facilitates expression of several genes. CFTR has been reported to regulate expression of RANTES which are chemokines mediating mucosal immunity [21]. Similarly, CFTR has also been reported to regulate MUC1 (mucins) and tyrosine kinase c-Src expression [22]. Role of mitochondrial dysfunction in CFTR became more confirmative by the finding of CFTR dependent regulation of two mitochondrial genes CISD1 and MT-ND4 [23,24]. CISD1 gene encodes for a protein with a CDGSH iron-sulfur domain and which is localized to the outer mitochondrial membrane whereas, MT-ND4 encodes for MT-ND4 mitochondrial Complex I (mtCx-I) subunit [23,24]. Down regulation of CISD1 has been reported in CF cells, similar results were reported when CFTR chloride channel activity was disrupted using chemical inhibitors [23]. Similar results of down regulation of MTND4 in CF cells or an inhibition of CFTR chloride transport have been reported [24].

Role of CFTR in regulating MT-ND4 also supports the initial observation of Shapiro et al regarding altered kinetics of NADH dehydrogenase enzyme of mitochondrial complex I [11,13]. MTND4 also known as mitochondrially encoded NADH dehydrogenase 4 encodes for NADH dehydrogenase 4 protein (ND4), which is one of the subunit of NADH dehydrogenase enzyme or complex I of mitochondrial respiratory chain [24]. In concurrence with the observation of down regulation of MT-ND4 gene expression in CF cells, reduction in mitochondrial complex I activity as well as altered mitochondrial membrane potential has been demonstrated $[25,26]$.

CISD1 proteins also named as mitoNEET have been reported to belong to the family of iron-sulfur $(2 \mathrm{Fe}-2 \mathrm{~S})$ proteins with $\mathrm{Fe}-\mathrm{S}$ domain comprising of CDGSH amino acid sequence [27-29]. mitoNEET proteins have implicated in regulating cellular iron and homeostasis of reactive oxygen species (ROS) [27-29]. Suppression of mitoNEET proteins has implicated to result in accumulation of iron, ROS and autophagy [27]. However role of CISD1 downregulation in CF still remain unclear, but it may be due altered mitochondrial function due to accumulated iron and ROS.

Another important function of mitochondria is $\mathrm{Ca}^{2+}$ buffering to regulate $\mathrm{Ca}^{2+}$ signalling. $\mathrm{Ca}^{2+}$ signalling is vital for many cellular functions and deregulation of it has been reported in CF. Deregulation of mitochondrial $\mathrm{Ca}^{2+}$ buffering resulting in increased $\mathrm{Ca}^{2+}$ accumulation compared to control has been reported in mitochondria obtained by fibroblast culture from CF patient in research reported in 1970's and 1980's [16-18]. A study done using F508del-CFTR airway epithelial cells have reported reduced $\mathrm{Ca}^{2+}$ levels in CF cells compared to control [26]. They have also reported alteration in mitochondrial morphology to more fragmented form in CF human tracheal gland cells compared to control [26]. Mitochondrial membrane potential has also been reported to be reduced in CF cells compared to control [26]. Another study published recently has reported role of F508delCFTR accumulation in endoplasmic reticulum (ER) in deregulation of $\mathrm{Ca}^{2+}$ signalling in $\mathrm{CF}$ [30]. They have reported that accumulation of F508del-CFTR in ER results in increased SERCA (Sarcoendoplasmic Reticulum $\mathrm{Ca}^{2+}$ Transport ATPase) pump activity on the other hand activity of PMCA (Plasma Membrane $\mathrm{Ca}^{2+}$ ATPase) gets reduced [30]. The possible role of increased CFTR/SERCA interaction and decreased CFTR/PMCA in CF cells in deregulation of $\mathrm{Ca}^{2+}$ homeostasis has been reported [30]. Increased $\mathrm{Ca}^{2+}$ retention in ER was reported due to F508del-CFTR accumulation and the effect was rescued following F508del-CFTR clearance [30]. Mitochondrial $\mathrm{Ca}^{2+}$ uptake has also been reported to increase in CF cells, however clearance of F508del-CFTR ER trafficking failed to restore normal mitochondrial $\mathrm{Ca}^{2+}$ levels [30].

Apart from transport of chloride ions role of CFTR in GSH transport has also been reported [31-33]. Altered GSH level in CF has been reported by the initial studies done in 1970's $[19,20]$. They have demonstrated higher, GSH reductase activity and total GSH levels in blood cells of CF patients compared to non CF [19]. Studies done during 1990's demonstrated GSH deficiency in CF samples. Decreased GSH levels have been reported in CF epithelial lining fluid (ELF), apical medium obtained from CFTR-deficient cell culture [32,34] moreover, transfection of normal CFTR has been reported to result in increased GSH [32]. On the similar line study done using CFTR knockout (CFTR$\mathrm{KO})$ mice demonstrated decreased GSH levels in ELF, whereas activity of glutathione reductase and glutathione peroxidase were elevated in CFTR-KO lung tissue $[33,35]$.

Oxidative stress due to defective CFTR together with dysregulation of antioxidative effect due to decreased GSH level results in CF disease progression and its pathological hallmarks. Mitochondrial electron transport system is one of the major sources of ROS generation [36] and GSH plays an antioxidative role. Mitochondria do not produce GSH and thus are dependent on GSH uptake from the cytosol. Decreased levels of mitochondrial GSH have been reported in the lungs of CFTR-KO mice and CFTR-deficient human lung epithelial cells; mitochondrial oxidative stress was also increased [33]. Another study reports depleted mitochondrial GSH levels in CF impairs functioning 
of electron transport chain (complex I) and restoring of GSH levels by GSH monoethyl ester (GSH-EE) restores electron transport chain functioning and mitochondrial membrane potential [35]. GSH-EE was also reported to re-establish normal levels of Interleukin-8 (IL8) in CF cells [37].

\section{Conclusion}

A mutation in the CFTR protein hampers its biosynthetic processing resulting in its proteolytic degradation in ER [38]. Proteolytic degradation of CFTR results in disrupted intracellular ion transport, oxidative stress, imbalance in redox homeostais and resulting in pathological features of the disease like increased sodium and chloride levels in sweat, viscous mucous secretion, infection, inflammation etc. Mutated CFTR protein has been reported to affect mitochondrial structure as well as function. CFTR has also been reported to regulate expression of few mitochondrial genes. Functioning of mitochondrial electron transport chain, $\mathrm{Ca}^{2+}$ homeostasis, redox balance, GSH levels all have been reported to be distressed in CF. Thus further exploration of mechanism underlying regulation of mitochondrial structure and function by CFTR gene may open possible avenues for CF therapeutics.

\section{References}

1. Zielenski J, Rozmahel R, Bozon D, Kerem B, Grzelczak Z, et al. (1991) Genomic DNA sequence of the cystic fibrosis transmembrane conductance regulator (CFTR) gene. Genomics 10: 214-228.

2. Tsui LC, Buchwald M, Barker D, Braman JC, Knowlton R, et al. (1985) Cystic fibrosis locus defined by a genetically linked polymorphic DNA marker. Science 230: 1054-1057.

3. Welsh MJ, Smith AE (1993) Molecular mechanisms of CFTR chloride channel dysfunction in cystic fibrosis. Cell 73: 1251-1254.

4. Riordan JR, Rommens JM, Kerem BS, Alon N, Rozmahel R, et al. (1989) Identification of the cystic fibrosis gene: cloning and characterization of complementary DNA. Science 245: 1066-1073.

5. Rommens JM, lannuzzi MC, Kerem B, Drumm ML, Melmer G, et al. (1989) Identification of the cystic fibrosis gene: chromosome walking and jumping. Science 245: 1059-1065.

6. Ashlock MA, Olson ER (2011) Therapeutics development for cystic fibrosis: a successful model for a multisystem genetic disease. Annu Rev Med 62: 107-125.

7. Sheppard DN, Welsh MJ (1999) Structure and function of the CFTR chloride channel. Physiol Rev 79: S23-45.

8. Protasevich I, Yang Z, Wang C, Atwell S, Zhao X, et al. (2010) Thermal unfolding studies show the disease causing F508del mutation in CFTR thermodynamically destabilizes nucleotide-binding domain 1. Protein Science 19: $1917-1931$

9. Shapiro BL (1989) Evidence for a mitochondrial lesion in cystic fibrosis. Life Sci 44: 1327-1334.

10. Feigal RJ, Shapiro BL (1979) Mitochondrial calcium uptake and oxygen consumption in cystic fibrosis. Nature 278: 276-277.

11. Shapiro BL, Feigal RJ, Lam LF (1979) Mitrochondrial NADH dehydrogenase in cystic fibrosis. Proc Natl Acad Sci U S A 76: 2979-2983.

12. Shapiro BL (1988) Mitochondrial dysfunction, energy expenditure, and cystic fibrosis. Lancet 2: 289

13. Shapiro BL, Lam LF, Feigal RJ (1982) Mitochondrial NADH dehydrogenase in cystic fibrosis: enzyme kinetics in cultured fibroblasts. Am J Hum Genet 34 : 846-852.

14. Dechecchi MC, Girella E, Cabrini G, Berton G (1988) The Km of NADH dehydrogenase is decreased in mitochondria of cystic fibrosis cells. Enzyme 40: 45-50.

15. Battino M, Rugolo M, Romeo G, Lenaz G (1986) Kinetic alterations of cytochrome-c oxidase in cystic fibrosis. FEBS Lett 199: 155-158.

16. Feigal RJ, Shapiro BL (1979) Altered intracellular calcium in fibroblasts from patients with cystic fibrosis and heterozygotes. Pediatr Res 13: 764-768.
17. Shapiro BL, Lam LF (1987) Intracellular calcium in cystic fibrosis heterozygotes. Life Sci 40: 2361-2366.

18. von Ruecker AA, Bertele R, Harms HK (1984) Calcium metabolism and cystic fibrosis: mitochondrial abnormalities suggest a modification of the mitochondria membrane. Pediatr Res 18: 594-599.

19. Shapiro BL, Smith QT, Warwick WJ (1973) Red cell glutathione and glutathione reductase in cystic fibrosis. Proc Soc Exp Biol Med 144: 181-183.

20. Shapiro BL, Smith QT, Warick WJ (1975) Serum glutathione reductase and cystic fibrosis. Pediatr Res 9: 885-888.

21. Estell K, Braunstein G, Tucker T, Varga K, Collawn JF, et al. (2003) Plasma membrane CFTR regulates RANTES expression via its C-terminal PDZ interacting motif. Mol Cell Biol 23: 594-606.

22. González-Guerrico AM, Cafferata EG, Radrizzani M, Marcucci F, Gruenert D et al. (2002) Tyrosine kinase c-Src constitutes a bridge between cystic fibrosis transmembrane regulator channel failure and MUC1 overexpression in cystic fibrosis. Journal of Biological Chemistry 277: 17239-17247.

23. Taminelli GL, Sotomayor V, Valdivieso AG, Teiber ML, Marín MC, et al. (2008) CISD1 codifies a mitochondrial protein upregulated by the CFTR channel. Biochem Biophys Res Commun 365: 856-862.

24. Valdivieso AG, Marcucci F, Taminelli G, Guerrico AG, Alvarez S, et al. (2007) The expression of the mitochondrial gene MT-ND4 is downregulated in cystic fibrosis. Biochem Biophys Res Commun 356: 805-809.

25. Valdivieso AG, Clauzure M, Marín MC, Taminelli GL, Copiz MMM, et al. (2012) The mitochondrial complex I activity is reduced in cells with impaired Cystic Fibrosis Transmembrane Conductance Regulator (CFTR) function. PloS one 7: e48059.

26. Antigny F, Girardin N, Raveau D, Frieden M, Becq F, et al. (2009) Dysfunction of mitochondria $\mathrm{Ca} 2+$ uptake in cystic fibrosis airway epithelial cells. Mitochondrion 9: 232-241.

27. Tamir S, Paddock ML, Darash-Yahana-Baram M, Holt SH, Sohn YS, et al (2015) Structure-function analysis of NEET proteins uncovers their role as key regulators of iron and ROS homeostasis in health and disease. Biochim Biophys Acta 1853: 1294-1315.

28. Bai F, Morcos F, Sohn YS, Darash-Yahana M, Rezende CO, et al. (2015) The Fe-S cluster-containing NEET proteins mitoNEET and NAF-1 as chemotherapeutic targets in breast cancer. Proc Natl Acad Sci U S A 112: 3698-3703.

29. Wiley SE, Murphy AN, Ross SA, van der Geer P, Dixon JE (2007) MitoNEET is an iron-containing outer mitochondrial membrane protein that regulates oxidative capacity. Proc Natl Acad Sci U S A 104: 5318-5323.

30. Philippe R, Antigny F, Buscaglia P, Norez C, Becq F, et al. (2015) SERCA and PMCA pumps contribute to the deregulation of $\mathrm{Ca} 2+$ homeostasis in human $\mathrm{CF}$ epithelial cells. Biochimica et Biophysica Acta (BBA)-Molecular Cell Research 1853: 892-903.

31. Linsdell P, Hanrahan JW (1998) Glutathione permeability of CFTR. Am J Physiol 275: C323-326.

32. Gao L, Kim KJ, Yankaskas JR, Forman HJ (1999) Abnormal glutathione transport in cystic fibrosis airway epithelia. American Journal of PhysiologyLung Cellular and Molecular Physiology 277: L113-L118.

33. Velsor LW, Kariya C, Kachadourian R, Day BJ (2006) Mitochondrial oxidative stress in the lungs of cystic fibrosis transmembrane conductance regulator protein mutant mice. American Journal of Respiratory Cell and Molecular Biology 35: 579-586.

34. Roum JH, Buhl R, McElvaney NG, Borok Z, Crystal RG (1993) Systemic deficiency of glutathione in cystic fibrosis. J Appl Physiol (1985) 75: 2419-2424.

35. Velsor LW, Heeckeren AV, Day BJ (2001) Antioxidant imbalance in the lungs of cystic fibrosis transmembrane conductance regulator protein mutant mice. American Journal of Physiology-Lung Cellular and Molecular Physiology 281 L31-L38.

36. Turrens JF (2003) Mitochondrial formation of reactive oxygen species. J Physiol 552: 335-344.

37. Kelly-Aubert M, Trudel S, Fritsch J, Nguyen-Khoa T, Baudouin-Legros M, et al (2011) GSH monoethyl ester rescues mitochondrial defects in cystic fibrosis models. Human Molecular Genetics 20: 2745-2759.

38. Ward CL, Omura S, Kopito RR (1995) Degradation of CFTR by the ubiquitinproteasome pathway. Cell 83: 121-127. 